
\title{
BReserch S Suare \\ Urine miRNA Signature as a Potential Non-invasive Diagnostic and Prognostic Biomarker in Cervical Cancer
}

\section{Mehreen Aftab}

Amity University

Satish Poojari

Amity University

Vaishnavi Seshan

Vardhman Mahavir Medical College \& Safdarjung Hospital

\section{Sachin Kumar}

All India Institute of Medical Sciences

\section{Pallavi Agarwal}

Amity University

\section{Simran Tandon}

Amity University

\section{Vijay Zutshi}

Vardhman Mahavir Medical College \& Safdarjung Hospital

Bhudev Das ( $\sim$ bcdas@amity.edu )

Amity Institute of Molecular Medicine \& Stem Cell Research (AIMMSCR), Amity University Campus, Sector-125, Noida, 201313, Uttar Pradesh, India

\section{Research Article}

Keywords: MicroRNAs, serum, plasma and other body fluids, HPV infection, survival outcome

Posted Date: December 7th, 2020

DOl: https://doi.org/10.21203/rs.3.rs-115644/v1

License: (c) (1) This work is licensed under a Creative Commons Attribution 4.0 International License.

Read Full License 


\section{Urine miRNA signature as a potential non-invasive diagnostic and prognostic biomarker in cervical cancer}

Mehreen Aftab ${ }^{1}$, Satish Poojari ${ }^{1}$, Vaishnavi Seshan ${ }^{2}$, Sachin Kumar ${ }^{3}$, Pallavi Agarwal ${ }^{1}$, Simran Tandon ${ }^{1}$, Vijay Zutschi ${ }^{2}$, Bhudev C. Das ${ }^{1 *}$

${ }^{1}$ Amity Institute of Molecular Medicine \& Stem Cell Research (AIMMSCR), Amity University Campus, Sector-125, Noida, 201313, Uttar Pradesh, India

${ }^{2}$ Department of Gynecology and Obstetrics, Safdarjung Hospital, New Delhi-110029, India

${ }^{3}$ Depatment of Medical Oncology, Dr. B R Ambedkar Institute Rotary Cancer Hospital, All India Institute of Medical Sciences, Ansari Nagar, New Delhi-110029, India

*Correspondence and materials request should be addressed to B.C.D (bcdas@ amity.edu)

\section{ABSTRACT}

MicroRNAs as cancer biomarkers in serum, plasma and other body fluids are often used but analysis of miRNA in urine is limited. We investigated the expression of selected miRNAs in the paired urine, serum, cervical scrape and tumor tissue specimens from the women with cervical precancer and cancer with a view to identify if urine miRNAs could be used as reliable noninvasive biomarkers for an early diagnosis and prognosis of cervical cancer. Expression of three oncomiRs (miR-21, miR-199a, and miR-155-5p) and three tumor suppressors (miR-34a, miR-145, and miR-218) in cervical pre-cancer, cancer and normal controls including cervical cancer cell lines were analyzed using qRT-PCR. The expression of miRNAs was correlated with various clinicopathological parameters, including HPV infection and survival outcome. We observed a significant overexpression of the oncomiRs and the downregulation of tumor suppressor miRNAs. A combination of miR-145-5p, miR-218-5p, and miR-34a-5p in urine yielded $100 \%$ sensitivity and $92.8 \%$ specificity in distinguishing precancer and cancer patients from healthy controls. The expression of miR-34a-5p and miR-218-5p were found to be independent prognostic factors for overall survival of cervical cancer patients. We conclude that the evaluation of specific miRNA expression in non-invasive urine samples may serve as reliable biomarker for early detection and prognosis of cervical cancer. 


\section{INTRODUCTION}

Cervical cancer is the fourth most common cancer in women with an estimated 300,000 deaths and 600,000 new cases reported in 2018[1]. Vast majority ( 85\%) of cervical cancer cases are detected in the less developed nations, where it accounts for $\sim 12 \%$ of all female cancers [2]. Biologically, cervical cancer begins in the form of pre-malignant lesions referred to as squamous intraepithelial lesions (SILs) or cervical intraepithelial lesions (CINs) that progress to carcinoma through different stages in a period of 10 to 20 years [3]. Persistent infection of high-risk (HR) human papillomaviruses (HPVs) has been considered as the principal cause for cervical cancer [4]. Currently, most commonly available HPV vaccines (Cervarix and Gardasil) provide protection against only two most common HR-HPV types, -16 and -18. But not to other HR-HPV types [5,6]. Available evidence suggests that there is a long gap between HPV infection and development of low-grade lesions that progress to invasive cancer $[7,8]$. This window period provides a unique opportunity for understanding molecular pathways involved in cervical tumorigenesis and identification of cancer biomarker(s) for early diagnosis and prognosis which may result in the better management of cervical cancer.

MicroRNAs (miRNAs) are small, non-protein coding RNAs of 18-25 nucleotides involved in posttranscriptional gene silencing by binding to 3' untranslated region (UTR) of its target messenger RNA (mRNA). Since miRNAs are known to regulate diverse cellular and biological processes, including cell proliferation, differentiation, inflammation, immune modulation, and programmed cell death, any deregulation in their expression may result in the initiation and progression of cancer. Overexpressed miRNAs may function as oncogenes by negatively regulating the expression of tumor suppressor genes, while downregulated miRNAs function as tumor suppressors by negatively regulating the oncogenic mRNAs. Differential expression of oncogenic and tumor suppressor miRNAs is proposed as a candidate biomarker in many cancers [9.10]. Apart from their detection in tumor tissues, several studies have also delineated the role of miRNAs derived from liquid biopsy samples, including plasma and serum, in cervical cancer [11-14]. However, it is thought that urine could be a better non-invasive liquid biopsy sample than blood because of the homeostasis mechanisms inside the body which may reflect changes in the blood [15]. Also, urine can be easily self-collected recurrently, in relatively large volumes. Although most studies have focused on the identification of biomarkers in blood, the ease of availability of 
urine and the high rate of patient compliance suggest that it could provide a promising source for the screening of patients for cervical cancer if the results match with that of the blood or tumor tissue. Earlier it has been demonstrated that HPV can be detected in urine with high sensitivity and specificity [16-18]. However, at present, the studies on expression profiling of miRNAs in urine for their use as potential biomarker(s) for screening, early detection, and prognosis of cervical cancer are lacking. The stage-specific detection of cervical cancer using non-invasive urine sampling could pave the way for large scale screening studies in a controlled manner. Therefore, the present study was designed to profile selected miRNA expression in urine and to compare the same with those of paired serum, cervical scrapes or cervical tumor tissues in order to validate specific urine miRNA(s) as biomarkers. After a comprehensive literature survey on their targets related for this, we used a shortlisted panel of six miRNAs (miR-199a-5p, miR-21-5p, miR-1555p, miR-145-5p, miR-34a-5p, and miR-218-5p) associated with HPV induced cervical cancer.

\section{RESULTS}

\section{Prevalence of HPV infection in urine compared with paired cervical scrapes and biopsies in cervical pre-cancer, cancer and control}

A noninvasive urine sampling has been utilized to establish if urine can serve as an alternative clinical material for reliable detection of HPV and other sensitive biomarkers such as miRNA expression for early detection of cervical cancer. Therefore, in the present study, paired urine samples, cervical scrapes and tissue biopsies from 50 subjects each of pre-cancer, cancer along with adjacent normal tissues and normal subjects were collected and subjected to detection and genotyping of HPV types 16 and 18 which are the most common high-risk oncogenic HPV types worldwide and are present in over $90 \%$ of carcinomas of Indian women. The analysis of HPV infection and its genotyping was conducted by PCR using consensus and HPV type-specific primers.

PCR-based detection revealed that $30(60 \%)$ out of a total of 50 urine samples of pre-cancer lesions were HPV positive. Out of these 50 precancer urine samples, 17 LSIL and 33 HSIL had 4 (8\%) and $26(52 \%)$ HPV positives, respectively. HPV infection was detected in the cervical scrape of $35 / 50(70 \%)$ of precancer lesions with $14 \%$ of LSIL and $56 \%$ of HSIL found to be HPV positive. However, in control samples $(n=50)$, only $3(6 \%)$ urine samples and $4(8 \%)$ normal cervical 
samples were found to be HPV positive (Table 1). Subsequently, HPV type specific PCRs performed in these cases to determine the prevalence of two most prevalent HR-HPV types 16 and 18 in urine samples revealed the presence of HPV16 DNA sequence in 28 out of $50(56 \%)$ cervical pre-cancer cases [4(8\%) of LSIL and $24(48 \%)$ of HSIL] whereas in cervical scrapes it was 32 out of 50 (64\%) with 4 (8\%), LSIL and 28 (56\%), HSIL and 2 (4\%) were positive both in urine and cervical scrapes controls. No sample was found positive for HPV type 18.

In cancer cases $(\mathrm{n}=50), \mathrm{HPV}$ positivity was detected in 40 out of $50(80 \%)$ urine samples and 36 out of $40(90 \%)$ tumor tissue biopsies. HR-HPV type 16 was the most frequently detected HPV type in the urine (74\%) and tumor biopsies (85\%) of cervical cancer patients. Interestingly, HPV18 infection was found only in 2 (4\%) each in urine and tumor biopsy of cancer patients. We also collected paired adjacent normal tissues as controls from 30 cervical cancer cases which revealed the presence of HPV16 infection in only 4 (8\%) cases. Interestingly, none of the paired adjacent normal tissues were positive for HPV 18 infection (Table 1).

When we examined the distribution of HPV prevalence with respect to differentiation histopathological grades, we obtained HPV positivity in 23 (46\%), 10 (20\%), 7 (14\%) in urine, while $16(40 \%), 10(25 \%), 10$ (25\%) in tumor tissue of WDSCC, MDSCC and PDSCC, respectively (Table 1). The HPV status along with histopathological grades and differentiation status of the tumors are presented in Table 1. HPV positivity in urine, cervical scrapes, and tissue biopsies was also correlated with clinical stage of the tumors (Table 1). HPV was found present in urine of 10 (20\%) cases of stage I, 17 (34\%) of stage II, 15 (30\%) of stage III and $3(6 \%)$ in stage IV. Majority of HPV positive tumors had HPV16 genotype regardless of tumor grade and sample types (Table 1). The urine samples of all stage I and II cervical cancer patients was HPV positive, while in paired tissue biopsies it was found that 8 out of 10 cases of stage I and 15 out of 17 of stage II patients were HPV positive. Further, in the urine samples, 15 out of 18 of stage III and 3 out of 5 of stage IV cervical cancer patients were found to be HPV positive, while in paired tissue biopsies, all stage III and stage IV patients were HPV positive (Table 1).

When the results of three paired clinical/biological sample viz. urine, cervical scrapes and tumor biopsies were compared for the presence of HPV infection, there was no significant difference rather very good correlation of results. 
Expression profiling of selected six miRNAs in paired urine, serum, cervical scrape, and tissue biopsy in control, cervical pre-cancer and cancer

We analyzed the expression of six miRNAs (miR-21-5p, miR-199a-5p, miR-155-5p, miR-145-5p, miR-34a-5p and miR-218-5p) in aforementioned samples using qRT-PCR (Table 2). A total of 460 samples were analyzed for miRNA expression (in 150 urine and serum samples collected from 50 subjects each of normal, cervical pre-cancer, and cancer, 50 cervical scrapes from pre-cancer patient, 40 cervical scrapes from normal controls, 40 tumor tissue biopsies and 30 paired adjacent normal tissues from cervical cancer patients). We found a significant upregulation of miR-21-5p, miR-199a-5p, and miR-155-5p and downregulation of miR-145-5p, miR-34a-5p, and miR-218-5p in urine and paired serum, cervical scrape or tumor biopsies (Table 2 and Figure 1) as well as in cervical cancer cell lines (Table S1). Compared to paired adjacent normal tissues, the expression of miR-21-5p ( $\mathrm{FC}=3.4)$, miR-199a-5p ( $\mathrm{FC}=2.2)$, and miR-155-5p ( $\mathrm{FC}=2.6)$ was upregulated while that of miR-145-5p ( $F C=0.12)$, miR-34a-5p $(F C=0.14)$, and miR-218-5p $(F C=0.13)$ was downregulated in tissue biopsies of cervical cancer patients (Table 2; Figure 1c and 1d; Figure S1d). Similar results were also obtained for cervical scrapes from cervical pre-cancer cases when compared with cytological normal samples (Table 2; Figure 1a and 1b, and Figure S1c).

To determine whether there is a similar trend of miRNAs expression in urine as in tumor biopsies, the correlation of all six miRNAs expression in urine and paired tumor biopsies was calculated using Pearson's correlation coefficient. Interestingly, the expression of all six miRNAs significantly and positively correlated in urine and paired tumor biopsies of cervical cancer cases (Figure S2) suggesting that miRNA expression profile of urine might accurately reflects to that in tumor cells. 


\section{Correlation of miRNA expression with HPV infection status}

We checked the expression of all six miRNAs in HPV16 positive cervical cancer cell line SiHa, HPV18 positive cervical cancer cell line HeLa, and HPV negative cervical carcinoma cell line C33A. Similar to the results in paired urine, serum, cervical scrape and tissue biopsies, qRT-PCR assay showed a marked upregulation in the expression of miR-21-5p, miR-155-5p, and miR-199a$5 p$ and downregulation in the expression of miR-34a-5p, miR-218-5p, and miR-145-5p in HPV positive and negative cell lines (Table S1). The results of expression of upregulated miRNA in paired samples of urine, serum, cervical scrapes and tumor biopsies of HPV positive and negative infection showed no significant difference rather perfect correlation of results (Figure 2).

When we compared the miRNA expression in HPV16 positive and negative samples of cervical pre-cancer and cervical cancer patients, we found that the expression of miR-145-5p was significantly lower in HPV16 positive urine samples of cervical pre-cancer (Figure S3a) and

cervical cancer patients (Figure S3d). Further, the expression of miR-21-5p was significantly higher in the serum of HPV16 positive than HPV negative cervical pre-cancer patients (Figure S3b). Moreover, the expression of miR-21-5p, miR-155-5p, and miR-199a-5p was significantly higher in HPV16 positive than HPV16 negative tissue biopsies of cervical cancer patients (Table S2; Figure S3f).

\section{Correlations of miRNA expression level with clinicopathological characteristics of cervical pre-cancer and cancer.}

The expression of all six miRNAs in urine, serum, cervical scrape, and tissue biopsies was correlated with various clinicopathological characteristics and known risk factors for cervical cancer. Except miR-34a-5p in the urine and miR-34a-5p and miR-199a-5p in the serum, the expression of all other miRNAs significantly correlated with the age of the cervical pre-cancer patients (Table S3). The expression of miR-21-5p (in urine and serum), miR-145-5p (in urine), miR-155-5p (in serum), and miR-199a-5p (in urine and serum) correlated with the age of marriage of cervical pre-cancer patients (Table S3). Further, the expression of miR-21-5p (in urine and serum), miR-145-5p (in serum), and miR-199a-5p (in urine and serum) correlated with the parity of cervical pre-cancer patients (Table S3). 
In cervical cancer patients, the expression of miR-21-5p (in serum), miR-218-5p (in urine and serum), miR-155-5p (in urine and serum), and miR-199a-5p (in serum) correlated with the age (Table S3). Further, the expression of miR-21-5p, miR-155-5p, and miR-199a-5p in urine and serum and the expression of miR-145-5p and miR-218-5p in serum correlated with the age of marriage of cervical cancer patients (Table S3). The expression of all 6 miRNAs in urine and serum significantly correlated with the stage of cervical cancer (Table S3).

In cervical scrapes of pre-cancer patients, we found a significant correlation between the expression of miR-145-5p, miR-218-5p, miR-34a-5p, and miR-199a-5p with age (Table S3). Further, the expression of miR-21-5p and miR-155-5p correlated with the age of marriage while that of miR-21-5p, miR-155-5p, and miR-199a-5p significantly correlated with parity (Table S3). In tissue biopsies of cervical cancer patients, except miR-145-5p and miR-34a-5p, the expression of all other miRNAs significantly correlated with the age (Table S4). Additionally, the expression of all 6 miRNAs in tumor biopsies significantly correlated with the stage of cervical cancer patients (Table S4).

\section{Diagnostic performance of miRNAs for cervical cancer as revealed by ROC analysis}

To evaluate the diagnostic utility of urinary and tissue miRNAs for cervical cancer, ROC analysis was performed (Table 3; Figure 3 and S4). ROC analysis demonstrated that urinary miRNAs may serve as useful non-invasive biomarkers for discriminating cervical pre-cancer and cancer from healthy controls. miR-21-5p exhibited the best AUC (0.971) with a sensitivity and specificity of $88 \%$ and $98 \%$, respectively (Table 3). In an attempt to improve the diagnostic performance of miRNAs, we constructed a binary logistic regression model to evaluate the performance of the combined use of 3 or all 6 urinary miRNAs together. Interestingly, a combination of miR-145-5p, miR-218-5p and miR-34a-5p yielded a 100\% sensitivity and 92.8\% specificity for distinguishing cervical cancer patients from healthy controls (Table 3). As evident from Table 3, the diagnostic performance of miRNA expression evaluated in tumor biopsies was better than urinary miRNAs. A combination of 3 miRNAs (miR-21-5p, miR-155-5p, and miR-199-5p) as well as all 6 miRNAs

yielded a 100\% sensitivity and specificity for distinguishing cervical cancer patients from healthy controls (Table 3). The absolute sensitivities and specificities achieved after combining multiple miRNAs indicate that detection of a combination of miRNAs in liquid biopsy sample like urine 
can prove to be a useful biomarker for the early diagnosis of cervical pre-malignant lesions and for reducing the incidence of cervical cancer and better management.

\section{Prognostic value of miRNA expression levels in cervical cancer}

We constructed a prognostic signature by integrating the expression profiles of six miRNAs and corresponding estimated regression coefficient. To evaluate the ability of these miRNAs as a signature to predict prognosis, we first carried out a univariate Cox proportional hazards regression analysis to evaluate the correlation between each of the six miRNAs and the survival outcome. The analysis revealed a significant correlation of mir-155-5p, miR-21-5p, miR-218-5p, and mir34a-5p expression with the overall survival (OS) (Table 4). A combination of 3 upregulated miRNA signature and 3 downregulated miRNA signatures also significantly correlated with OS (Table 4). We also performed multivariate Cox regression analysis to find independent prognostic power of aforementioned miRNAs within the context of several common clinical parameters, including age at diagnosis, age of marriage, parity, HPV infection, and tumor stage. The multivariate analysis revealed that miR-34a-5p $(\mathrm{HR}=4.418 ; \mathrm{p}=0.01), \operatorname{miR}-218-5 \mathrm{p} \quad(\mathrm{HR}=0.332$; $\mathrm{p}=0.049)$, and HPV16 status $(\mathrm{HR}=1.428 ; \mathrm{p}=0.019)$ were independent prognostic factors of OS (Table 4). Further, a combination of 3 upregulated miRNA signature $(H R=5.956 ; p<0.0001)$ and 3 downregulated miRNA signatures $(\mathrm{HR}=0.194 ; \mathrm{p}<0.0001)$ also retained their significance as independent prognostic factors of OS (Table 4).

Further, we performed the Kaplan Meier survival analysis to examine the potential prognostic power of the six miRNAs (Figure 4). Notably, different expression levels of miR-155-5p, miR21-5p, miR-199a-5p, miR-218-5p, miR-34a-5p have led to significantly different survival rates (log-rank test $\mathrm{p}$-value $<0.0001, \mathrm{p}<0.001, \mathrm{p}<0.0001, \mathrm{p}=0.007$ and $\mathrm{p}=0.006$, respectively), while no significant survival differences were observed between the high and low expression set of miR145-5p (log-rank test p-value $=0.09$ ). We divided into a high expression group and a low expression group relative to the cut off expression level of these six miRNAs across all samples.

\section{Target gene prediction and functional analysis of miRNAs}

To further understand the functions of all 6 miRNAs, the online target gene prediction was performed using three prediction algorithms: miRDB, Target Scan, and microT-CDS. As evident from Figure S5, all 6 miRNAs can potentially regulate the expression of a large number of genes. 
The number of overlapping targets for miR-21-5p, miR-155-5p, miR-199a-5p, miR-34a-5p, miR145-5p, and miR-218-5p were 274, 208, 234, 568, 1708, and 508, respectively (Figure S5). Subsequently, pathway enrichment analysis was performed on all overlapping potential target genes of six miRNAs to investigate their potential biological functions (Figure S6A). The Kyoto Encyclopedia of Genes and Genomes (KEGG) pathway was significantly enriched in PI3K-AKT pathway, microRNA in cancer, pathways in cancer and mTOR signaling pathways (Figure S6B), whereas the REACTOME pathway was significantly enriched in signaling transduction, RNA polymerase II transcription pathway, and immune system (Figure S6B). Since most of these pathways are well known for their role in tumor cell proliferation, angiogenesis, apoptosis, tumor cell migration and invasion, we can emphasize the involvement of all 6 miRNAs in the development and progression of cervical cancer.

\section{DISCUSSION}

HPV infection is the major risk factors for cervical cancer and it may take 10-20 years after getting HPV infection to develop invasive cervical cancer. Hence, cervical cancer represents a suitable model not only to dissect molecular determinants of cancer initiation and progression but also to identify cancer biomarkers for early diagnosis and prognosis of cervical cancer. Identification of cancer biomarker using non-invasive methods is highly useful for easy screening, monitoring treatment response and risk assessment. Hence, we explored the clinical utility of urinary miRNAs as non-invasive diagnostic and prognostic biomarkers in cervical pre-cancer and cervical cancer patients. Urine generally contains a mixture of circulating cells and local cells from epithelial wall of the cervix, vaginal discharge, menstrual blood, and cervical exfoliated cells containing HPV and miRNAs in the urine might be secreted from any of the origins mentioned above. We observed a significant positive correlation of miRNA expression in urine with paired serum, cervical scrapes or tumor tissue of cervical cancer patients indicating that the analysis of miRNA or any genetic or epigenetic alterations in urine may offer a better and alternative biological material to tissue biopsy for an easy diagnosis and prognosis of cervical cancer.

In the present study, we evaluated the expression of 6 important miRNAs (miR-21-5p, miR-199a5p, miR155-5p, miR-34a-5p, miR-145-5p, and miR-218-5p) selected based on results of several previous studies $[19,20]$. Some of these miRNAs are also found to be associated with HR-HPV infection in cervical pre-cancer and cancer [21,22]. The expression level of six urinary miRNAs 
was found to be significantly altered (Upregulated: miR-21-5p, miR-199a-5p, and miR-155-5p; Downregulated: miR-145-5p, miR-34a-5p, and miR-218-5p) in our cohort of cervical pre-cancer and cancer patients as compared to that of healthy controls. The expression level of 6 selected miRNAs in urine exhibited similar trends as in paired tissue biopsies, cervical scrapes and serum of precancer and cancer patients. The fold-change in the expression level of miR-21, miR-155 and miR-199a was higher in cervical scrapes than in serum and urine of precancer cases; but certainly, significantly higher than controls. On the contrary, the fold-change in the expression level of miR145, miR-34a and miR-218 was higher in urine than in serum and cervical scrapes and the difference in fold-change among various sample types was found to be statistically significant. The upregulation of urinary miR-21, miR-155, and miR-199a among precancer and cancer cases align with earlier studies which demonstrated overexpression of these miRNAs in cervical scrape and tumor biopsy in cervical cancer by invasive method [23-25]. The correlation between these oncomiRs expression in paired urine, serum, cervical scrapes and tumor biopsy suggests that urinary miRNAs could be used as a potential biomarker for investigating cervical abnormalities.

The downregulation of urinary miR-145, miR-34a and miR-218 in cervical precancer and cancer when compared with normal healthy control is in line with an earlier study which showed that downregulation of these three miRNAs in the cervical scrape and tumor tissue correlate well with malignant transformation [26,27].

To evaluate the diagnostic value of urinary miRNAs in cervical cancer, ROC analysis was performed for individual miRNA [28,29]. Among the six miRNAs studied, miR-21-5p was found to be the best diagnostic biomarker $(\mathrm{AUC}=0.971)$ for differentiating the cervical cancer patients with the $88 \%$ sensitivity and 98\% specificity. Further, ROC analysis of a combination of all 6 urinary miRNAs revealed absolute sensitivity and specificity of miRNA signature in discriminating cervical pre-cancer and cancer patients from normal individuals. Such high sensitivity and specificity of the urinary miRNA signature indicates that they could serve as a potential biomarker for the early diagnosis of cervical pre-malignant lesions and cancer. If urinary miRNA of pre cancer with distinctly differential ROC value particularly for low grade squamous intraepithelial lesion (LSIL), can be detected early and treated effectively, leading to the reduction of cervical cancer incidence. 
We also analyzed the clinical utility of miRNAs as prognostic biomarkers by correlating their expression with overall survival (OS) of cervical cancer patients. We found that miR-34a-5p and miR-218-5p are significantly associated with OS of cervical cancer patients in a multivariate analysis indicating their utility as independent prognostic biomarkers. A combination of 3 upregulated miRNA signature and 3 downregulated miRNA signature appears to be independent prognostic factors of OS suggesting that multi-miRNA-based model may provide more powerful information for predicting the prognosis of cervical cancer patients.

Previous studies have demonstrated that these miRNAs are crucial for the initiation, progression, and metastasis of cervical cancer by regulating various pathways, including cancer cell proliferation, differentiation, apoptosis, adhesion, cell cycle arrest, migration, and invasion [3340]. miR-21 promoted cell proliferation and invasion by targeting programmed cell death 4 (PDCD4) in cervical cancer[22], while miR-155 did the same by targeting SOCS1, C/EBP $\beta$, and SHIP1[24,41,42] Lee et al(2010) demonstrated upregulation of miR-199a in cervical cancer $[25,43]$ and is considered as a potential therapeutic target for cervical cancer[44].The expression of miR-218, which can target $L A M B 3$ [27], was downregulated in cervical cancer following with HR-HPV infection. The expression of miR-145 was also found to be downregulated in cervical cancer tissues and cell lines and was associated with tumor size, lymph node metastasis, HPV16 infection, and poor prognosis[58,59].Further,miR-34a has been reported to be downregulated in cervical cancer cell lines HeLa, SiHa, C4I, C33a, and CaSki affecting Notch and Jagged1 proteins[45]. Together these findings indicate that these six miRNAs may serve as potential target(s) for effective diagnosis, therapy of cervical cancer.

We also examined the significance of HPV infection as such, as it is the principal etiological agent responsible for transformation of normal cervical epithelium to pre-neoplastic cervical intraepithelial neoplasia that subsequently undergoes malignant transformation to invasive cervical cancer. However, the exact mechanism is still unclear. Proteins encoded by E6 and E7 genes of HR- HPVs bind to and degrade 553 and Rb proteins respectively [46,47]. HPV-associated the six miRNAs are directly or indirectly regulated by HPV E5-E7 proteins, play an important role in the initiation and progression of cervical cancer $[21,48]$. The expression of miR-21-5p, miR199-5p, and miR-155-5p was found to be significantly increased in HPV16 positive than HPV16 negative cervical cancer patients. These results suggested that the aberrant expression of these 
miRNAs might be the consequence of HR-HPV induced cervical carcinogenesis $[23,48-51]$. HRHPV encoded E6 and E7 oncoproteins are the major oncogenic drivers of cellular transformation. During virus induced cellular transformation, it also interacts with many other signaling pathways, including the miRNA pathways [46,47,52,53].The expression of miR-199a-5p,miR-218-5p,miR145-5p, and miR-34a-5p was also reported to be regulated by HPV[48,27,20]. The upregulation of miR-21 in cervical cancer was observed in association with the dysregulation of DROSHA, a critical enzyme in the miRNA biogenesis pathway, for which the expression is suggested to be modulated by HPV16 E6/E7 oncoproteins[21,40].HPV oncoproteins E6 and E7 were found to suppress miR-145-5p expression[43,54].

Furthermore, we screened the target genes of these six miRNAs and predicted the enrichment pathways and biological functions of target genes using bioinformatics tools as miRNAs, have been considered as the master modulators of multiple biological and pathological pathways during cancer development. To gain a deeper insight into the molecular functions of these miRNAs, we predicted the target genes and analyzed the related signaling pathways and GO annotations. We observed that these six miRNAs could regulate several key signaling pathways, including MAPK pathways, AMPK pathways, focal adhesion, cGMP-PKG, Wnt/ $\beta$-catenin, and mTOR signaling pathways. Accumulating evidence has demonstrated that activation of MAPK pathway is important in cervical cancer progression, invasion, and metastasis[52,53]. Yung et al.(2013) and Kwan et al (2013) reported that activation of AMP-activated protein kinase (AMPK), a metabolic sensor, hinders cervical cancer cell growth through reducing the expression of FOXM1 and blocking the DVL3 mediated Wnt/ $\beta$-catenin signaling pathway[55,56]. Also, the transformation of human keratinocytes with HPV oncoproteins E6 and E7 requires activation of the Wnt/ $\beta$ catenin pathways and $\beta$-catenin relocate from cytoplasm to nucleus as the cervical cancer progress [52]. This activation may serve as a screening tool in HPV-infected population to detect malignant progression and prognosis [52]. Moreover, it has been well established that the $\mathrm{PI} 3 \mathrm{~K} / \mathrm{Akt} / \mathrm{mTOR}$ signaling pathway plays a crucial role in cervical cancer development and inhibition of mTOR kinase activity suppress tumor growth [53]. Therefore, further investigations in larger sample size are required to validate these observations as it can provide new avenues for developing effective therapeutic interventions in cervical cancer.

\section{MATERIALS AND METHODS}




\section{Collection and processing of biological samples}

For this prospective study, we recruited 150 subjects comprising of 50 subjects each of cervical pre-cancer, cervical cancer, and healthy controls from the outpatient department of Gynecology and Obstetrics, Safdarjung Hospital, New Delhi. Cervical scrapes from pre-cancer lesions were collected from low or high grade squamous intraepithelial lesion (LSIL/HSIL) patients. Tumor tissue biopsies were collected from patients undergoing surgical resection or radical hysterectomy for invasive cervical cancer. From all patients, urine was collected before the collection of cervical scrapes or biopsies. The study was approved by Institutional Ethics Committee of Amity University, Uttar Pradesh (AUUP), Noida (India) and Institutional Ethics Committee of V.M. Medical college \& Safdarjung Hospital, New Delhi (India). Written informed consent was obtained from all subjects. The study was carried out in accordance with the guidelines and principles of the Helsinki Declaration.

Thirty to fifty $\mathrm{ml}$ of midstream urine was collected from all the subjects and stored at $4{ }^{\circ} \mathrm{C}$ for 2hours before process. Urine sample from each patient was divided into two parts and centrifuged at 3,000rpm for $10 \mathrm{~min}$. The resulting pellet was washed twice with sterile $1 \mathrm{X}$ phosphate buffer saline (PBS pH 7.4). For the detection of HPV, pellet was suspended in 1ml of 1XPBS (pH 7.4) and stored at $-20^{\circ} \mathrm{C}$. For RNA isolation, pellet was mixed with $1 \mathrm{ml}$ of TRIzol reagent (Invitrogen, Carlsbad, CA, USA) and stored at $-80^{\circ} \mathrm{C}$. Cervical scrapes obtained from cervical pre-cancer patients and healthy controls, by scraping the ectocervix with a wooden spatula or cytobrush, and tissue biopsies obtained from cervical cancer patients were collected in 1XPBS (pH 7.4) and TRIzol reagent (Invitrogen, Carlsbad, CA, USA). Serum was prepared from $2 \mathrm{ml}$ of venous blood, which was allowed to clot for $20 \mathrm{~min}$ at room temperature followed by centrifugation at 3,000 rpm for 10 minutes.

\section{Cell Culture}

Human cervical cancer cell lines (SiHa, HeLa, and C33a) were cultured in Dulbecco's Modified Eagle Medium (DMEM, Sigma Aldrich) supplemented with 10\% heat-inactivated fetal bovine serum (FBS, Sigma Aldrich), 2 mM L-glutamine (Sigma Aldrich) and penicillin and streptomycin $100 \mathrm{U} / \mathrm{mL}$ and $100 \mu \mathrm{g} / \mathrm{mL}$ (Sigma Aldrich), respectively, at $37^{\circ} \mathrm{C}$ with $5 \% \mathrm{CO}_{2}$.

\section{HPV detection and genotyping}


Genomic DNA was extracted from urine samples, cervical scrapes and tumor biopsies using standard proteinase $\mathrm{K}$ digestion, phenol/chloroform extraction and ethanol precipitation as described previously[18,57,58,32]. The quantity and quality of DNA was assessed using agarose gel electrophoresis and by UV spectrophotometry (Nanodrop, Thermo Fisher Scientific). HPV detection was done by PCR using a pair of HPVL1 consensus primers MY09 and MY11 that gave an amplicon of $450 \mathrm{bp}$ (Table S5). HPV genotyping was done by PCR using type-specific primers for predominant HPV types 16 and 18 . PCR was performed in a $25 \mu 1$ reaction mixture containing 100ng DNA, $10 \mathrm{mM}$ Tris- $\mathrm{HCl}$ (pH8.4), $50 \mathrm{mM} \mathrm{KCl}, 1.5 \mathrm{mM} \mathrm{MgCl}, 125 \mathrm{mM}$ of each dNTPs (Thermo Fisher Scientific), 5pmol of oligonucleotide primers and 0.5U Taq DNA polymerase. $\beta$ globin gene was used as an internal control. Rest of the method was standard procedures followed in the lab [18].

\section{RNA extraction and cDNA synthesis}

Total RNA extraction from urine, cervical scrapes, tissue biopsies, and cervical cancer cell lines was done using TRIzol reagent (Invitrogen, Carlsbad, CA, USA) according to the manufacturer's instruction. Total RNA was isolated from $250 \mu 1$ serum using TRIzol LS reagent (Invitrogen, Carlsbad, CA, USA) according to manufacturer's instructions. RNA concentration and purity were determined by Nanodrop (Thermo Fisher Scientific). cDNA synthesis was done from total RNA using High Capacity cDNA synthesis kit (Thermo Fisher Scientific). Briefly, miRNAs were reverse transcribed using 500ng of total RNA and pools of miRNA-specific stem loop primers following manufacturer's instructions (Table S6). The cDNA was stored in $-20^{\circ} \mathrm{C}$ and later used for quantitative reverse transcription polymerase chain reaction (qRT-PCR).

\section{Analysis of miRNA expression using qRT-PCR}

qRT-PCR reactions were performed separately for RNA extracted from urine, serum, cervical scrape, tissue biopsies, and cell lines with their corresponding normal controls using SYBR Green (Applied Biosystems, USA). snRNA U6 was used as a reference control. The threshold cycle data were determined using the default threshold settings. Same concentration of cDNA was used for all miRNA analysis in order to maintain consistency and same efficiency. The reaction conditions were $10 \mathrm{~min}$ at $95^{\circ} \mathrm{C}$, followed by 45 cycles of $95^{\circ} \mathrm{C}$ for $15 \mathrm{~s}$ and $60^{\circ} \mathrm{C}$ for $1 \mathrm{~min}$. All experiments were run in triplicate, along with a negative and a positive control. The fold change of miRNA 
expression for each sample in relation to normal control was calculated based on the threshold cycle $(\mathrm{CT})$ value using the following formula: Relative Quantification $(\mathrm{RQ})=2^{-\Delta \Delta \mathrm{CT}}[59,60]$.

\section{Target gene prediction and enrichment analysis}

The potential target genes of candidate miRNAs were predicted through three different online algorithm miRDB (http://www.mirdb.org/miRDB)[61,62],TargetScanv7.1 (http://www.targetscan.org/) and DIANA microT-CDS web Server v 5.0 (http://www.microrna.gr/webServer)[63]. To further support the consistency of the bioinformatics analysis, the overlapping target genes from three online tools of each miRNA were identified using a Venn diagram (https://omics.pnl.gov/software/venn-diagram-plotter)[64,65]. The gene ontology enrichment was conducted by The ShinyGO v0.60: Gene Ontology Enrichment Analysis (http://bioinformatics.sdstate.edu/go/)[66] and pathway enrichment of overlapping target genes was conducted through The Database for Annotation, Visualization and Integrated Discovery (DAVID v6.7) (http://david.nicfcrf.gov)[25,67] bioinformatics online tool. $\mathrm{p}<0.05$ was set as the cut-off criteria.

\section{Statistical analysis}

Data were analyzed using Graph pad prism statistics software program version 5 and IBM SPSS statistics software program version 26. The data was exhibited as the mean \pm SD (standard deviation). Mann-Whitney test as well as student's t-test was employed to compare the significant mean difference of miRNAs expression correlation between two groups. Kruskal-Wallis test was done to check the significant mean difference between different sampling groups. The correlation analysis to assess the relationship between miRNA expression and clinical features was performed by Pearson's correlation coefficient. The diagnostic performance of miRNAs was checked by plotting receiver operator characteristic (ROC) curve. The prognostic value was assessed by employing Kaplan-Meier curve and Long-rank method for each differentially expressed miRNA. The miRNAs that were significantly associated with OS were identified as prognostic miRNAs and then subjected to a binary logistic regression analysis. Subsequently, a prognostic miRNA signature was constructed for calculating a risk score for each cervical cancer patient. With the miRNA signature, cervical cancer patients were classified into high risk (high expression) and low risk (low expression) groups using the median risk score. Kaplan-Meier method was employed for 
evaluating the differences in patients' survival between low risk and high-risk groups. Cox proportional hazards regression analysis was carried out for evaluating the impact of miRNAs on survival time and clinical survival data of the patients. Patients who had poor chances of survival were the ones having high-risk score [67].

\section{CONCLUSION}

The six urinary miRNA signatures (miR-21-5p, miR-155-5p, miR-199a-5p, miR-145-5p, miR218-5p and miR-34a-5p) were identified and characterized with oncogenic and tumor suppressor potential, and can serve as superior diagnostic and prognostic biomarkers with high sensitivity and specificity, cervical pre-cancer and cancer. However, prospective studies comparing miRNA signatures with cytology and HPV are required to further validate the present findings before the use of urinary miRNAs as reliable non- invasive biomarkers for cervical and other cancers. 
Acknowledgements: The authors are thankful to the Laboratory colleagues and Staff of Amity Institute of Molecular Medicine and Stem Cell Research, Amity University, Uttar Pradesh and Outpatient department of Gynecology and Obstetrics, Safdarjung Hospital, New Delhi, India.

Author contributions: M.A. performed sample collection, conducted all experiments and data collection, compilation and drafting of the manuscript. S. P. assisted with the RT-qPCR experimentation followed by statistical analysis. V.S. and V.Z. assisted in provide clinical samples and their pathology and histopathology details of the patients. S.K., S.T. and P.A. carried out statistical analysis, and helped in critical reading of the manuscript. B.C.D conceptualized and designed the study, checked analysis and did interpretation of data, supervised and executed the entire study, critically revised and edited and communicated the manuscript.

Competing interests: The authors declare no competing interests.

Additional information: Supplementary information is available for this paper at journal website

Author information: Correspondence and request for materials should be addressed to B.C.D (bcdas@amity.edu) 


\section{REFERENCES}

1. Bray F, Ferlay J, Soerjomataram I, Siegel RL, Torre LA, Jemal A. Global cancer statistics 2018: GLOBOCAN estimates of incidence and mortality worldwide for 36 cancers in 185 countries. CA Cancer J Clin. 2018;68(6):394-424. doi:10.3322/caac.21492

2. WHO | Human papillomavirus (HPV). WHO. 2018.

3. Rodríguez AC, Schiffman M, Herrero R, et al. Rapid clearance of human papillomavirus and implications for clinical focus on persistent infections. J Natl Cancer Inst. 2008;100(7):513-517. doi:10.1093/jnci/djn044

4. JM W, MV J, MM M, et al. Human Papillomavirus Is a Necessary Cause of Invasive Cervical Cancer Worldwide. J Pathol. 1999;189(1). doi:10.1002/(SICI)10969896(199909)189:1<12::AID-PATH431>3.0.CO;2-F

5. Montgomery MP, Dune T, Shetty PK, Shetty AK. Knowledge and Acceptability of Human Papillomavirus Vaccination and Cervical Cancer Screening among Women in Karnataka, India. J Cancer Educ. 2015;30(1):130-137. doi:10.1007/s13187-014-0745-4

6. Malik H, Khan FH, Ahsan H. Human papillomavirus: Current status and issues of vaccination. Arch Virol. 2014;159(2):199-205. doi:10.1007/s00705-013-1827-z

7. Schiffman M, Wentzensen N. Human papillomavirus infection and the multistage carcinogenesis of cervical cancer. Cancer Epidemiol Biomarkers Prev. 2013;22(4):553560. doi:10.1158/1055-9965.EPI-12-1406

8. Mighty KK, Laimins LA. The role of human papillomaviruses in oncogenesis. Recent Results Cancer Res. 2014;193:135-148. doi:10.1007/978-3-642-38965-8_8

9. Zhang L, Huang J, Yang N, et al. microRNAs exhibit high frequency genomic alterations in human cancer. Proc Natl Acad Sci U S A. 2006;103(24):9136-9141. doi:10.1073/pnas.0508889103

10. Lu J, Getz G, Miska EA, et al. MicroRNA expression profiles classify human cancers. Nature. 2005;435(7043):834-838. doi:10.1038/nature03702

11. Jia W, Wu Y, Zhang Q, Gao GE, Zhang C, Xiang Y. Expression profile of circulating microRNAs as a promising fingerprint for cervical cancer diagnosis and monitoring. Mol Clin Oncol. 2015;3(4):851-858. doi:10.3892/mco.2015.560

12. Nagamitsu Y, Nishi H, Sasaki T, Takaesu Y, Terauchi F, Isaka K. Profiling analysis of circulating microRNA expression in cervical cancer. Mol Clin Oncol. 2016;5(1):189-194. doi:10.3892/mco.2016.875

13. Jiang Y, Hu Z, Zuo Z, et al. Identification of Circulating MicroRNAs as a Promising Diagnostic Biomarker for Cervical Intraepithelial Neoplasia and Early Cancer: A MetaAnalysis. Biomed Res Int. 2020;2020. doi:10.1155/2020/4947381 
14. Xin F, Liu P, Ma CF. A circulating serum miRNA panel as early detection biomarkers of cervical intraepithelial neoplasia. Eur Rev Med Pharmacol Sci. 2016;20(23):4846-4851.

15. Campion CG, Sanchez-Ferras O, Batchu SN. Potential role of serum and urinary biomarkers in diagnosis and prognosis of diabetic nephropathy. Can J Kidney Heal Dis. 2017;4. doi:10.1177/2054358117705371

16. Das BC, Krishna VG, Sharma JK, Roy M, Luthra UK. Human Papillomavirus DNA in urine of women with preneoplastic and neoplastic cervical lesions. Lancet.

1992;340:1363-1420.

17. Das BC, Murthy NS, Sharma JK, et al. human papillomavirus and cervical cancer in indian women. Lancet. 1989;334(8674):1271. doi:10.1016/S0140-6736(89)91870-9

18. Prusty BK, Kumar A, Arora R, Batra S, Das BC. Human papillomavirus (HPV) DNA detection in self-collected urine. Int J Gynecol Obstet. 2005;90(3):223-227. doi:10.1016/j.ijgo.2005.06.004

19. Tornesello ML, Faraonio R, Buonaguro L, et al. The Role of microRNAs, Long Noncoding RNAs, and Circular RNAs in Cervical Cancer. Front Oncol. 2020;10. doi:10.3389/fonc. 2020.00150

20. González-Quintana V, Palma-Berré L, Campos-Parra AD, et al. MicroRNAs are involved in cervical cancer development, progression, clinical outcome and improvement treatment response (Review). Oncol Rep. 2016;35(1):3-12. doi:10.3892/or.2015.4369

21. Lajer CB, Garnæs E, Friis-Hansen L, et al. The role of miRNAs in human papilloma virus (HPV)-associated cancers: Bridging between HPV-related head and neck cancer and cervical cancer. Br J Cancer. 2012;106(9):1526-1534. doi:10.1038/bjc.2012.109

22. Bumrungthai S, Ekalaksananan T, Evans MF, et al. Up-regulation of MIR-21 is associated with cervicitis and human papillomavirus infection in cervical tissues. PLoS One. 2015;10(5). doi:10.1371/journal.pone.0127109

23. Farzanehpour M, Mozhgani SH, Jalilvand S, et al. Serum and tissue miRNAs: Potential biomarkers for the diagnosis of cervical cancer. Virol J. 2019;16(1):116. doi:10.1186/s12985-019-1220-y

24. Lao G, Liu P, Wu Q, et al. Mir-155 promotes cervical cancer cell proliferation through suppression of its target gene LKB1. Tumor Biol. 2014;35(12):11933-11938. doi:10.1007/s13277-014-2479-7

25. Zhu XL, Wen SY, Ai ZH, Wang J, Xu YL, Teng YC. Screening for characteristic microRNAs between pre-invasive and invasive stages of cervical cancer. Mol Med Rep. 2015;12(1):55-62. doi:10.3892/mmr.2015.3363

26. Tornesello ML, Faraonio R, Buonaguro L, et al. The Role of microRNAs, Long Noncoding RNAs, and Circular RNAs in Cervical Cancer. Front Oncol. 2020;10:150. 
doi:10.3389/fonc.2020.00150

27. Zhu L, Tu H, Liang Y, Tang D. MiR-218 produces anti-tumor effects on cervical cancer cells in vitro. World J Surg Oncol. 2018;16(1). doi:10.1186/s12957-018-1506-3

28. Mitchell PS, Parkin RK, Kroh EM, et al. Circulating microRNAs as stable blood-based markers for cancer detection. Proc Natl Acad Sci U S A. 2008;105(30):10513-10518. doi:10.1073/pnas.0804549105

29. Alhasan AH, Scott AW, Wu JJ, et al. Circulating microRNA signature for the diagnosis of very high-risk prostate cancer. Proc Natl Acad Sci U S A. 2016;113(38):10655-10660. doi:10.1073/pnas.1611596113

30. zur Hausen H. Human papillomaviruses and their possible role in squamous cell carcinomas. Curr Top Microbiol Immunol. 1977;78:1-30. doi:10.1007/978-3-642-66800$5 \_1$

31. Orth G, Croissant O. Papillomaviruses and human cancer. Bull Inst Pasteur. 1988;86(3):297-315. doi:10.1007/978-1-4615-3738-0_8

32. Das BC, Gopalkrishna V, Hedau S, Katiyar S. Cancer of the Uterine Cervix and Human Papillomavirus Infection Uterine Cervical Cancer. Vol 78.; 2000.

33. Khoury S, Tran N. Circulating microRNAs: Potential biomarkers for common malignancies. Biomark Med. 2015;9(2):131-151. doi:10.2217/BMM.14.102

34. Allegra A, Alonci A, Campo S, et al. Circulating microRNAs: New biomarkers in diagnosis, prognosis and treatment of cancer (Review). Int J Oncol. 2012;41(6):18971912. doi:10.3892/ijo.2012.1647

35. Hussain S, Nasare V, Kumari M, et al. Perception of human papillomavirus infection, cervical cancer and HPV vaccination in North Indian population. PLoS One. 2014;9(11). doi:10.1371/journal.pone.0112861

36. Zhang Z, Wang J, Wang X, Song W, Shi Y, Zhang L. MicroRNA-21 promotes proliferation, migration, and invasion of cervical cancer through targeting TIMP3. Arch Gynecol Obstet. 2018;297(2):433-442. doi:10.1007/s00404-017-4598-z

37. Kang HW, Wang F, Wei Q, et al. miR-20a promotes migration and invasion by regulating TNKS2 in human cervical cancer cells. FEBS Lett. 2012;586(6):897-904. doi:10.1016/j.febslet.2012.02.020

38. Zhang Z, Wang J, Li J, Wang X, Song W. MicroRNA-150 promotes cell proliferation, migration, and invasion of cervical cancer through targeting PDCD4. Biomed Pharmacother. 2018;97:511-517. doi:10.1016/j.biopha.2017.09.143

39. Wang N, Zhou Y, Zheng L, Li H. MiR-31 is an independent prognostic factor and functions as an oncomir in cervical cancer via targeting ARID1A. Gynecol Oncol. 
2014;134(1):129-137. doi:10.1016/j.ygyno.2014.04.047

40. Wang J yan, Chen L juan. The role of miRNAs in the invasion and metastasis of cervical cancer. Biosci Rep. 2019;39(3). doi:10.1042/BSR20181377

41. Higgs G, Slack F. The multiple roles of microRNA-155 in oncogenesis. J Clin

Bioinforma. 2013;3(1):17. doi:10.1186/2043-9113-3-17

42. Faraoni I, Antonetti FR, Cardone J, Bonmassar E. miR-155 gene: A typical multifunctional microRNA. Biochim Biophys Acta - Mol Basis Dis. 2009;1792(6):497505. doi:10.1016/j.bbadis.2009.02.013

43. Wang X, Tang S, Le SY, et al. Aberrant expression of oncogenic and tumor-suppressive microRNAs in cervical cancer is required for cancer cell growth. PLoS One. 2008;3(7). doi:10.1371/journal.pone.0002557

44. Wei D, Wang W, Shen B, et al. MicroRNA-199a-5p suppresses migration and invasion in oral squamous cell carcinoma through inhibiting the EMT-related transcription factor SOX4. Int J Mol Med. 2019;44(1):185-195. doi:10.3892/ijmm.2019.4174

45. MicroRNA-34a suppresses invasion through downregulation of Notch1 and Jagged1 in cervical carcinoma and choriocarcinoma cells | Carcinogenesis | Oxford Academic. https://academic.oup.com/carcin/article/31/6/1037/2630015. Accessed May 28, 2020.

46. Yugawa T, Kiyono T. Molecular mechanisms of cervical carcinogenesis by high-risk human papillomaviruses: Novel functions of E6 and E7 oncoproteins. Rev Med Virol. 2009;19(2):97-113. doi:10.1002/rmv.605

47. Narisawa-Saito M, Kiyono T. Basic mechanisms of high-risk human papillomavirusinduced carcinogenesis: Roles of E6 and E7 proteins. Cancer Sci. 2007;98(10):1505-1511. doi:10.1111/j.1349-7006.2007.00546.x

48. Park S, Eom K, Kim J, et al. MiR-9, miR-21, and miR-155 as potential biomarkers for HPV positive and negative cervical cancer. BMC Cancer. 2017;17(1):658.

doi:10.1186/s12885-017-3642-5

49. Nadu T. Differential mirna expression profiling in HPV-16. 2019;5(869). doi:10.26479/2019.0502.65

50. Pardini B, De Maria D, Francavilla A, Di Gaetano C, Ronco G, Naccarati A. MicroRNAs as markers of progression in cervical cancer: A systematic review. BMC Cancer. 2018;18(1):696. doi:10.1186/s12885-018-4590-4

51. Ye J, Cheng XD, Cheng B, Cheng YF, Chen XJ, Lu WG. MiRNA detection in cervical exfoliated cells for missed high-grade lesions in women with LSIL/CIN1 diagnosis after colposcopy-guided biopsy. BMC Cancer. 2019;19(1). doi:10.1186/s12885-019-5311-3

52. Bello JOM, Nieva LO, Paredes AC, Gonzalez AMF, Zavaleta LR, Lizano M. Regulation 
of the Wnt $\beta$-catenin signaling pathway by human papillomavirus E6 and E7 oncoproteins. Viruses. 2015;7(8):4734-4755. doi:10.3390/v7082842

53. Howley PM, Münger K, Romanczuk H, Scheffner M, Huibregtse JM. Cellular targets of the oncoproteins encoded by the cancer associated human papillomaviruses. Princess Takamatsu Symp. 1991;22:239-248. http://www.ncbi.nlm.nih.gov/pubmed/1668886. Accessed May 30, 2020.

54. Gunasekharan V, Laimins LA. Human Papillomaviruses Modulate MicroRNA 145 Expression To Directly Control Genome Amplification. J Virol. 2013;87(10):6037-6043. doi:10.1128/jvi.00153-13

55. Yung MMH, Chan DW, Liu VWS, Yao KM, Ngan HYS. Activation of AMPK inhibits cervical cancer cell growth through AKT/FOXO3a/FOXM1 signaling cascade. BMC Cancer. 2013;13:327. doi:10.1186/1471-2407-13-327

56. Kwan HT, Chan DW, Cai PCH, et al. AMPK Activators Suppress Cervical Cancer Cell Growth through Inhibition of DVL3 Mediated Wnt/ $\beta$-Catenin Signaling Activity. PLoS One. 2013;8(1). doi:10.1371/journal.pone.0053597

57. Gupta A, Arora R, Gupta S, et al. Human papillomavirus DNA in urine samples of women with or without cervical cancer and their male partners compared with simultaneously collected cervical/penile smear or biopsy specimens. Clin Virol. 2006;37:190-194.

58. Sehgal A, Gupta S, Parashari A, Sodhani P, Singh V. Urine HPV-DNA detection for cervical cancer screening: prospects and prejudices. J Obstet Gynaecol. 2009;29(7):583589. doi:10.1080/01443610903061736

59. Thakur S, Grover RK, Gupta S, Yadav AK, Das BC. Identification of specific miRNA signature in paired sera and tissue samples of Indian women with triple negative breast cancer. PLoS One. 2016;11(7):158946. doi:10.1371/journal.pone.0158946

60. Shishodia G, Shukla S, Srivastava Y, et al. Alterations in microRNAs miR-21 and let-7a correlate with aberrant STAT3 signaling and downstream effects during cervical carcinogenesis. Mol Cancer. 2015;14(1):116. doi:10.1186/s12943-015-0385-2

61. Wang X. miRDB: A microRNA target prediction and functional annotation database with a wiki interface. RNA. 2008;14(6):1012-1017. doi:10.1261/rna.965408

62. miRDB: an online resource for microRNA target prediction and functional annotations | Nucleic Acids Research | Oxford Academic. https://academic.oup.com/nar/article/43/D1/D146/2437436. Accessed May 28, 2020.

63. Paraskevopoulou M, ... GG-N acids, 2013 undefined. DIANA-microT web server v5. 0: service integration into miRNA functional analysis workflows. academic.oup.com. https://academic.oup.com/nar/article-abstract/41/W1/W169/1100417. Accessed May 28, 2020 . 
64. Yi Y, Liu Y, Wu W, Wu K, Zhang W. Reconstruction and analysis of circRNA-miRNA-mRNA network in the pathology of cervical cancer. Oncol Rep. 2019;41(4):2209-2225. doi:10.3892/or.2019.7028

65. Liu SS, Chan KKL, Chu DKH, et al. Oncogenic microRNA signature for early diagnosis of cervical intraepithelial neoplasia and cancer. Mol Oncol. 2018;12(12):2009-2022. doi:10.1002/1878-0261.12383

66. Xijin Ge S, Jung D. ShinyGO: a graphical enrichment tool for ani-mals and plants. doi:10.1101/315150

67. Liu C, Yu Z, Huang S, et al. Combined identification of three miRNAs in serum as effective diagnostic biomarkers for HNSCC. EBioMedicine. 2019;50:135-143. doi:10.1016/j.ebiom.2019.11.016

\section{FIGURE LEGENDS}

Figure 1: Comparative differential expression of six miRNAs in urine, serum, cervical scrape, and tissue biopsies derived from healthy controls, pre-cancer and cancer. (a \& c) The miRNA expression level of upregulated miRNAs (miR-21-5p, miR-155-5p, miR-199a-5p) and (b \& d) down regulated miRNAs (miR-145-5p, miR218 -5p, and miR-34a-5p). Urine and serum samples were taken from pre-cancer and cervical cancer patients and compared to samples from healthy controls. In case of cervical scrape, the samples were derived from pre-cancer patients compared to samples from healthy volunteers, while in case of tissue biopsies, the samples were derived from cancer patient compared to samples from adjacent non-malignant tissues. ${ }^{*} \mathrm{p} \leq 0.01$, ns (nonsignificant).

Figure 2: Expression levels of miR-21-5p, miR-155-5p, miR-199a-5p, miR-145-5p, miR-218-5p, and miR-34a-5p in HPV16-positive (a \& c) and HPV16-negative (b \& d) urine, serum, and cervical scrape samples of cervical pre-cancer and in HPV16-positive (e \& g) and HPV16-negative (f \& h) urine, serum, and tissue biopsies of cervical cancer patients .

Figure 3: Receiver operating characteristics (ROC) plots of miRNA expression in urine sample of cervical cancer patients. ROC plots evaluating diagnostic potential of (a) miR-21-5p, (b) miR155-5p, (c) miR-199a-5p, (d) combined ROC plots of three upregulated miRNAs (miR-21-5p, miR-155-5p, and miR-199a-5p), (e) miR-218-5p, (f) miR-145-5p, (g) miR-34a-5p, (h) combined ROC plots of three downregulated miRNAs (miR-218-5p, miR34a-5p, and miR-145-5p), and (1) combined ROC plots of all six miRNAs.

Figure 4: The correlation of miRNA expression with overall survival of cervical cancer patients calculated using Kaplan Meier curve and Log-rank test. The patients were stratified into high expression and low expression group according to median of each miRNA. (a) miR-21-5p, (b) miR-199a-5p, (c) miR-155-5p, (d) miR-218-5p, (e) miR-145-5p, (f) miR-34a-5p, (g) three 
upregulated miRNA signatures, and (h) three downregulated miRNA signatures. The patients were stratified into high risk group and low risk group based on median.

Figure S1: Differential expression of six miRNAs in urine, serum, cervical scrape, and tissue biopsies derived from healthy controls, pre-cancer and cervical cancer patients. The miRNA expression level of miR-21-5p, miR-155-5p, miR-199a-5p, miR-145-5p, miR218 -5p, and miR34a-5p in (a) urine, (b) serum, (c) cervical scrape, and (d) tissue biopsies. Urine and serum samples were taken from pre-cancer and cervical cancer patients and compared to samples from healthy controls. In case of cervical scrape, the samples were derived from pre-cancer patients compared to samples from healthy volunteers, while in case of tissue biopsies, the samples were derived from cancer patient compared to samples from adjacent non-malignant tissues. ${ }^{*} \mathrm{p} \leq 0.01,{ }^{* * *} \mathrm{p} \leq 0.001$.

Figure S2: Correlation of expression pattern of miRNAs in cervical tissue biopsies and paired urine using Pearson's correlation coefficient. Pearson's correlation scatter plots for the correlation between the fold-change expression levels of (a) miR-21-5p, (b) miR-155-5p, (c) miR-199a-5p, (d) miR-145-5p, (e) miR-34a-5p, and (f) miR-218-5p in tissue biopsies and paired urine of cervical cancer patients.

Figure S3: Expression levels of miR-21-5p, miR-155-5p, miR-199a-5p, miR-145-5p, miR-2185p, and miR-34a-5p in HPV16-positive and HPV16-negative urine, serum, and cervical scrape samples of cervical pre-cancer (a-c) and urine, serum, and tissue biopsies of cervical cancer patients (d-f).

Figure S4: (a-f) Differential expression of six miRNAs in pre-cancer, cancer and normal urine, and the ROC analyses of six miRNA detection. Box plot of miRNA expression in urine (upper: miR-21-5p, miR-155-5p and miR-199a-5p; lower: miR-145-5p, miR-218-5p and miR-34a-5p is normalized to U6. The lines inside the boxes indicate the medians. The boxes mark the interval between the 25 th and 75 th percentiles. The whiskers indicate the interval between and outside the 10th and 90th percentiles. A statistically significant difference is determined by Kruskal-Wallis test with Dunn's multiple comparison post-hoc test, normal urine.

Figure S5: The target gene prediction and function analyses. The Venn diagrams represent the overlapping target genes of (a) miR-21-5p, (b) miR-155-5p, (c) miR-199a-5p, (d) miR-34a-5p, (e) miR-145-5p, and (f) miR-218-5p predicted by miRDB, Target Scan and DIANA microTCDS online analysis tools.

Figure S6A: Top 10 Gene ontology enrichment in (a) Biological, (b) Molecular, and (c) Cellular processes.

Figure S6B: Top 10 pathway enrichment analysis, including (a) KEGG and (b) REACTOME pathways. 


\section{TABLE LEGENDS}

Table 1: Clinicopathological characteristics and HPV status of cervical pre-cancer and cancer patients

Table 2: Relative expression level of miRNAs in paired urine, serum, cervical scrapes, and tissue biopsies

Table 3: Diagnostic performance of miRNAs in cervical cancer patients

Table 4: Univariate and multivariate Cox regression analysis to evaluate the prognostic value of individual miRNAs

Table S1: The expression of miRNAs in HPV positive and HPV negative cervical cancer cell line

Table S2: The expression of miRNAs according to HPV infection status in urine, serum, cervical scrape, and tissue biopsies derived from cervical pre-cancer and cancer patients

Table S3: The correlation of the expression of urine, serum and cervical scrape miRNAs with the clinicopathological parameters of cervical pre-cancer patients

Table S4: The correlation of the expression of urine and serum miRNAs with the clinicopathological parameters of cervical cancer patients

Table S5: Primers for amplification of human papilloma virus gene sequence

Table S6: MiRNA primer sequences used for Real-time PCR 
Table 1: Clinicopathological characteristics and HPV status of cervical pre-cancer and cancer patients

\begin{tabular}{|c|c|c|c|c|c|c|c|c|}
\hline & \multirow[t]{2}{*}{ Pathology } & \multirow{2}{*}{$\begin{array}{l}\text { Number } \\
\text { of } \\
\text { samples }\end{array}$} & \multicolumn{2}{|c|}{$\begin{array}{c}\text { Total HPV+ }(\text { HPV L1 } \\
\text { Consensus) }(\mathbf{n} / \%)\end{array}$} & \multicolumn{2}{|c|}{$\begin{array}{l}\text { HPV16 Positive } \\
(\mathbf{n} / \%)\end{array}$} & \multicolumn{2}{|c|}{$\begin{array}{l}\text { HPV18 } \\
(\mathbf{n} / \%)\end{array}$} \\
\hline & & & Urine & $\begin{array}{l}\text { Cervical } \\
\text { scrape/Biopsy }\end{array}$ & Urine & $\begin{array}{l}\text { Cervical } \\
\text { scrape/ } \\
\text { Biopsy }\end{array}$ & Urine & $\begin{array}{l}\text { Cervical } \\
\text { scrape/Biopsy }\end{array}$ \\
\hline Normal /Control & & 50 & $3(6 \%)$ & $4(8 \%)$ & $2(4 \%)$ & $2(4 \%)$ & 0 & 0 \\
\hline \multirow[t]{3}{*}{ Pre-cancer } & & 50 & $\begin{array}{l}30 \\
(60 \%)\end{array}$ & $35(70 \%)$ & $\begin{array}{l}28 \\
(56 \%)\end{array}$ & $32(64 \%)$ & 0 & 0 \\
\hline & LSIL & 17 & $4(8 \%)$ & $7(14 \%)$ & $4(8 \%)$ & $4(8 \%)$ & 0 & 0 \\
\hline & HSIL & 33 & $\begin{array}{l}26 \\
(52 \%)\end{array}$ & $28(56 \%)$ & $\begin{array}{l}24 \\
(48 \%)\end{array}$ & $28(56 \%)$ & 0 & 0 \\
\hline Cancer & & 50 & $\begin{array}{l}40 \\
(80 \%)\end{array}$ & $36 *(90 \%)$ & $\begin{array}{l}37 \\
(74 \%)\end{array}$ & $34 *(85 \%)$ & $\begin{array}{l}2 \\
(4 \%)\end{array}$ & $2(4 \%)$ \\
\hline \multirow[t]{3}{*}{$\begin{array}{l}\text { Histopathological } \\
\text { grading }\end{array}$} & WDSCC & 28 & $23(46 \%)$ & $16(40 \%)$ & $\begin{array}{l}20 \\
(40 \%)\end{array}$ & $14(35 \%)$ & 0 & 0 \\
\hline & MDSCC & 12 & $\begin{array}{l}10 \\
(20 \%)\end{array}$ & $10(25 \%)$ & $\begin{array}{l}10 \\
(20 \%)\end{array}$ & $10(25 \%)$ & 0 & 0 \\
\hline & PDSCC & 10 & $7(14 \%)$ & $10(25 \%)$ & $\begin{array}{l}7 \\
(14 \%)\end{array}$ & $10(25 \%)$ & 0 & 0 \\
\hline \multirow[t]{5}{*}{ Clinical stage } & & 50 & $\begin{array}{l}45 \\
(90 \%)\end{array}$ & $46(92 \%)$ & $\begin{array}{l}40 \\
(80 \%)\end{array}$ & $42(84 \%)$ & 0 & 0 \\
\hline & Stage I & 10 & $\begin{array}{l}10 \\
(20 \%)\end{array}$ & $8(16 \%)$ & $\begin{array}{l}8 \\
(16 \%)\end{array}$ & $8(16 \%)$ & 0 & 0 \\
\hline & Stage II & 17 & $\begin{array}{l}17 \\
(34 \%)\end{array}$ & $15(30 \%)$ & $\begin{array}{l}14 \\
(28 \%)\end{array}$ & $15(30 \%)$ & 0 & 0 \\
\hline & Stage III & 18 & $15(30 \%)$ & $18(36 \%)$ & $\begin{array}{l}15 \\
(30 \%)\end{array}$ & $15(30 \%)$ & $\begin{array}{l}1 \\
(2 \%)\end{array}$ & $1(2 \%)$ \\
\hline & Stage IV & 5 & $3(6 \%)$ & $5(10 \%)$ & $\begin{array}{l}3 \\
(6 \%)\end{array}$ & $4(8 \%)$ & $\begin{array}{l}1 \\
(2 \%)\end{array}$ & $1(2 \%)$ \\
\hline
\end{tabular}

*HPV detection conducted in 40 tissue biopsies

HPV: Human papillomavirus; SCC: Squamous cell carcinoma; LSIL: Low grade squamous intraepithelial lesions; HSIL: High grade squamous intraepithelial lesions; WDSCC: Well differentiated SCC; MDSCC: Moderately differentiated SCC; PDSCC: Poorly differentiated SCC 
Table 2: Relative expression level of miRNAs in paired urine, serum, cervical scrapes, and tissue biopsies

\begin{tabular}{|c|c|c|c|c|c|c|c|c|c|c|c|c|c|}
\hline Sample & Pathology & $\underset{(F C \pm S E)}{\operatorname{miR}-21-5 p}$ & p value & $\begin{array}{c}\text { miR-145-5p } \\
(\mathrm{FC} \pm \text { SE })\end{array}$ & p value & $\begin{array}{c}\operatorname{miR}-218-5 p \\
(\mathrm{FC} \pm \mathrm{SE})\end{array}$ & $\begin{array}{c}p \\
\text { value }\end{array}$ & $\begin{array}{c}\operatorname{miR}-34 a-5 p \\
(F C \pm S E)\end{array}$ & $\begin{array}{c}p \\
\text { value }\end{array}$ & $\begin{array}{c}\operatorname{miR}-155-5 p \\
(\mathrm{FC} \pm \mathrm{SE})\end{array}$ & $\begin{array}{c}p \\
\text { value }\end{array}$ & $\begin{array}{c}\operatorname{miR}-199 a-5 p \\
(F C \pm S E)\end{array}$ & $\begin{array}{c}p \\
\text { value }\end{array}$ \\
\hline \multirow{2}{*}{$\begin{array}{c}\text { Urine } \\
(\mathrm{n}=150)^{\mathrm{a}}\end{array}$} & $\begin{array}{c}\text { Pre-cancer } \\
(\mathrm{n}=50)\end{array}$ & $1.93 \pm 0.9$ & $<0.0001$ & $0.32 \pm 0.1$ & $<0.0002$ & $0.43 \pm 0.1$ & $<0.0003$ & $0.41 \pm 0.1$ & $<0.0001$ & $1.89 \pm 0.2$ & $<0.0001$ & $1.51 \pm 0.3$ & $<0.0001$ \\
\hline & $\begin{array}{l}\text { Cancer } \\
(\mathrm{n}=50)\end{array}$ & $2.42 \pm 0.1$ & $<0.0001$ & $0.24 \pm 0.5$ & $<0.0002$ & $0.32 \pm 0.4$ & $<0.0001$ & $0.36 \pm 0.2$ & $<0.0001$ & $2.27 \pm 0.20$ & $<0.0001$ & $1.73 \pm 0.1$ & $<0.0001$ \\
\hline \multirow{2}{*}{$\underset{(\mathrm{n}=150)^{\mathrm{a}}}{\text { Serum }}$} & $\begin{array}{l}\text { Pre-cancer } \\
\quad(\mathrm{n}=50)\end{array}$ & $2.10 \pm 0.20$ & $<0.0001$ & $0.15 \pm 0.01$ & $<0.0001$ & $0.34 \pm 0.09$ & $<0.0001$ & $0.27 \pm 0.2$ & $<0.0001$ & $1.72 \pm 0.1$ & $<0.0001$ & $1.81 \pm 0.2$ & $<0.0001$ \\
\hline & $\begin{array}{l}\text { Cancer } \\
(\mathrm{n}=50)\end{array}$ & $2.72 \pm 0.2$ & $<0.012$ & $0.18 \pm 0.01$ & $<0.0001$ & $0.26 \pm 0.03$ & $<0.008$ & $0.21 \pm 0.10$ & $<0.0001$ & $2.16 \pm 0.3$ & $<0.0001$ & $1.92 \pm 0.2$ & $<0.0001$ \\
\hline $\begin{array}{c}\text { Scrape } \\
(\mathrm{n}=90)^{\mathrm{b}}\end{array}$ & $\begin{array}{c}\text { Pre-cancer } \\
(\mathrm{n}=50)\end{array}$ & $2.3 \pm 0.1$ & $<0.0001$ & $0.13 \pm 0.1$ & $<0.0001$ & $0.28 \pm 0.02$ & $<0.0001$ & $0.23 \pm 0.01$ & $<0.0001$ & $1.94 \pm 0.2$ & $<0.0001$ & $2.02 \pm 0.3$ & $<0.0001$ \\
\hline $\begin{array}{c}\text { Tissue } \\
\text { biopsy } \\
(\mathrm{n}=70)^{\mathrm{c}}\end{array}$ & $\begin{array}{l}\text { Cancer } \\
(\mathrm{n}=40)\end{array}$ & $3.43 \pm 0.3$ & $<0.0001$ & $0.12 \pm 0.2$ & $<0.0001$ & $0.13 \pm 0.2$ & $<0.0001$ & $0.14 \pm 0.2$ & $<0.0001$ & $2.6 \pm 0.4$ & $<0.0001$ & $2.2 \pm 0.3$ & $<0.0001$ \\
\hline
\end{tabular}

${ }^{a}$ including 50 urine and 50 serum samples from normal controls; ${ }^{b}$ including 40 cervical scrape from normal controls; ${ }^{c}$ including 30 paired adjacent non-malignant normal tissue; FC: fold change; SE: standard error 
Table 3: Diagnostic performance of miRNAs in cervical cancer patients

\begin{tabular}{|c|c|c|c|c|c|c|}
\hline miRNAs & Source & Sensitivity & Specificity & AUC (95\% CI) & Cut-off & p value \\
\hline miR-21-5p & $\begin{array}{l}\text { urinary } \\
\text { miRNA }\end{array}$ & $88 \%$ & $98 \%$ & $0.971(0.957-0.985)$ & 1.912 & $<0.0001$ \\
\hline miR-155-5p & $\begin{array}{l}\text { urinary } \\
\text { miRNA }\end{array}$ & $86.7 \%$ & $91.7 \%$ & $0.719(0.816-0.907)$ & 0.8312 & $<0.0001$ \\
\hline miR-199a-5p & $\begin{array}{l}\text { urinary } \\
\text { miRNA }\end{array}$ & $93.8 \%$ & $88.3 \%$ & $0.831(0.869-0.945)$ & 0.8964 & $<0.0001$ \\
\hline miR-145-5p & $\begin{array}{l}\text { urinary } \\
\text { miRNA }\end{array}$ & $89.1 \%$ & $91.7 \%$ & $0.575(0.743-0.407)$ & 0.2387 & $<0.0001$ \\
\hline miR-218-5p & $\begin{array}{l}\text { urinary } \\
\text { miRNA }\end{array}$ & $67.7 \%$ & $60 \%$ & $0.609(0.729-0.482)$ & 0.4983 & $<0.0001$ \\
\hline miR-34a-5p & $\begin{array}{l}\text { urinary } \\
\text { miRNA }\end{array}$ & $83.3 \%$ & $72.7 \%$ & $0.888(0.781-1.00)$ & 0.0186 & $<0.0001$ \\
\hline $\begin{array}{l}\text { Combined three } \\
\text { downregulated miRNA } \\
\text { (miR-21-5p, miR-155- } \\
\text { 5p, miR-199-5p) } \\
\text { signature }\end{array}$ & $\begin{array}{l}\text { urinary } \\
\text { miRNAs }\end{array}$ & $100 \%$ & $78.9 \%$ & $0.942(0.868-1.00)$ & 0.5104 & $<0.0001$ \\
\hline $\begin{array}{lr}\begin{array}{l}\text { Combined } \\
\text { upregulated }\end{array} \text { three } \\
(\mathrm{miR}-145-5 \mathrm{p}, \mathrm{miR}-218- \\
\text { 5p,miR-34a-5p) } \\
\text { signature }\end{array}$ & $\begin{array}{l}\text { urinary } \\
\text { miRNAs }\end{array}$ & $100 \%$ & $92.8 \%$ & $0.969(0.912-1.000)$ & 0.7877 & $<0.0001$ \\
\hline $\begin{array}{l}\text { Combined ROC of all } \\
\text { six miRNAs }\end{array}$ & $\begin{array}{c}\text { urinary } \\
\text { miRNAs }\end{array}$ & $100 \%$ & $100 \%$ & $1.000(1.00-1.00)$ & -1.000 & $<0.0001$ \\
\hline miR-21-5p & \begin{tabular}{|c|}
$\begin{array}{c}\text { Tumor biopsy } \\
\text { miRNA }\end{array}$ \\
\end{tabular} & $98 \%$ & $85 \%$ & $0.993(0.975-1.000)$ & 1.182 & $<0.0001$ \\
\hline miR-155-5p & $\begin{array}{c}\text { Tumor biopsy } \\
\text { miRNA }\end{array}$ & $95 \%$ & $72.9 \%$ & $0.976(0.935-1.000)$ & 0.7562 & $<0.0001$ \\
\hline miR-199a-5p & $\begin{array}{c}\text { Tumor biopsy } \\
\text { miRNA }\end{array}$ & $96.5 \%$ & $92.7 \%$ & $0.978(0.964-1.000)$ & 1.302 & $<0.0001$ \\
\hline miR-145-5p & \begin{tabular}{|c|} 
Tumor biopsy \\
miRNA
\end{tabular} & $90 \%$ & $75 \%$ & $0.946(0.881-1.000)$ & 0.1546 & $<0.0001$ \\
\hline miR-218-5p & \begin{tabular}{|c|} 
Tumor biopsy \\
miRNA
\end{tabular} & $85 \%$ & $92.7 \%$ & $0.995(0.983-1.000)$ & 0.0652 & $<0.0001$ \\
\hline miR-34a-5p & \begin{tabular}{|c|}
$\begin{array}{c}\text { Tumor biopsy } \\
\text { miRNA }\end{array}$ \\
\end{tabular} & $73.2 \%$ & $91 \%$ & $0.826(0.713-0.940)$ & 0.2269 & $<0.0001$ \\
\hline $\begin{array}{l}\text { Combined three } \\
\text { downregulated miRNA } \\
\text { (miR-21-5p, miR-155- } \\
\text { 5p, miR-199-5p) } \\
\text { signature }\end{array}$ & $\begin{array}{c}\text { Tumor biopsy } \\
\text { miRNAs }\end{array}$ & $100 \%$ & $100 \%$ & $1.000(1.000-1.000)$ & -1.000 & $<0.0001$ \\
\hline $\begin{array}{l}\text { Combined three } \\
\text { upregulated miRNA } \\
\text { (miR-145-5p, miR-218- } \\
5 p, \text { miR-34a-5p) } \\
\text { signature } \\
\end{array}$ & $\begin{array}{c}\text { Tumor biopsy } \\
\text { miRNAs }\end{array}$ & $100 \%$ & $88.9 \%$ & $.932(.839-1.000)$ & 0.4202 & $<0.0001$ \\
\hline $\begin{array}{l}\text { Combined ROC of all } \\
\text { six miRNAs }\end{array}$ & $\begin{array}{c}\text { Tumor biopsy } \\
\text { miRNAs }\end{array}$ & $100 \%$ & $100 \%$ & $1.000(1.000-1.000)$ & -1.000 & $<0.0001$ \\
\hline
\end{tabular}


Table 4: Univariate and multivariate Cox regression analysis to evaluate the prognostic value of individual miRNAs

\begin{tabular}{|c|c|c|c|c|}
\hline miRNA & Univariat & alysis & Multivariate A & alysis* \\
\hline & HR (95\% CI) & p value & HR (95\% CI) & p value \\
\hline miR-155-5p & $\begin{array}{l}0.148(0.051- \\
0.431)\end{array}$ & $<0.001$ & $1.035(218-4.922)$ & 0.969 \\
\hline miR-21-5p & $\begin{array}{l}0.313(0.129- \\
0.759)\end{array}$ & 0.01 & $0.613(0.133-2.827)$ & 0.530 \\
\hline miR-199-5p & $\begin{array}{l}0.132(0.032- \\
0.557)\end{array}$ & 0.006 & $0.190(0.049-0.741)$ & 0.017 \\
\hline miR-218-5p & $\begin{array}{l}4.175(1.34- \\
13.0)\end{array}$ & 0.01 & $0.332(0.111-0.949)$ & 0.049 \\
\hline $\operatorname{miR}-34 a-5 p$ & $\begin{array}{l}6.248(1.44- \\
26.96)\end{array}$ & 0.01 & $\begin{array}{ll}4.418 & (1.410- \\
13.842)\end{array}$ & 0.01 \\
\hline $\operatorname{miR}-145-5 p$ & $\begin{array}{l}1.776(0.891- \\
3.518)\end{array}$ & 0.103 & $\begin{array}{l}2.748(0.675- \\
11.193)\end{array}$ & 0.158 \\
\hline Age of marriage & $\begin{array}{l}1.580(0.702- \\
4.516)\end{array}$ & 0.219 & $2.358(0.910-6.111)$ & 0.077 \\
\hline HPV 16 & $\begin{array}{l}0.225(0.103- \\
0.495)\end{array}$ & $<0.001$ & $1.428(0.503-3.654)$ & 0.019 \\
\hline Age & $\begin{array}{l}0.968(0.917- \\
6.031)\end{array}$ & 0.949 & $0.392(0.048-3.217)$ & 0.383 \\
\hline Parity & $\begin{array}{l}2.351(0.095- \\
1.308)\end{array}$ & 0.075 & $0.839(0.378-1.863$ & 0.677 \\
\hline T stage & $\begin{array}{l}1.159(0.367- \\
3.665)\end{array}$ & 0.802 & $0.442(0.082-2.372)$ & 0.341 \\
\hline $\begin{array}{l}\text { Three-up regulated } \\
\text { miRNA signatures (high } \\
\text { risk vs. low risk) }\end{array}$ & $\begin{array}{l}3.584(1.23- \\
10.410)\end{array}$ & 0.01 & $5.956(1.87-7.265)$ & $<0.0001$ \\
\hline $\begin{array}{l}\text { Three downregulated } \\
\text { miRNA signatures (high } \\
\text { risk vs. low risk) }\end{array}$ & $\begin{array}{l}0.239(0.072- \\
0.787)\end{array}$ & 0.01 & $0.194(0.065-0.564)$ & $<0.0001$ \\
\hline
\end{tabular}


HR: Hazard ratio; CI: Confidence interval

* Adjusted with age at diagnosis, age of marriage, parity, HPV infection, and tumor stage 
(a)

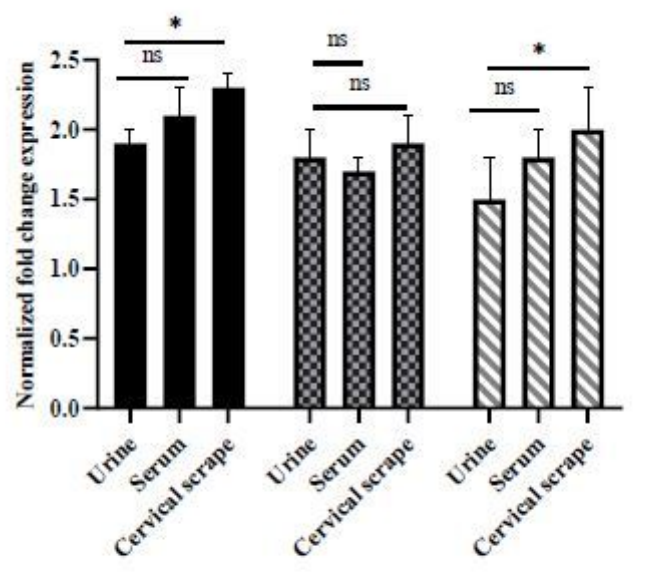

(c)

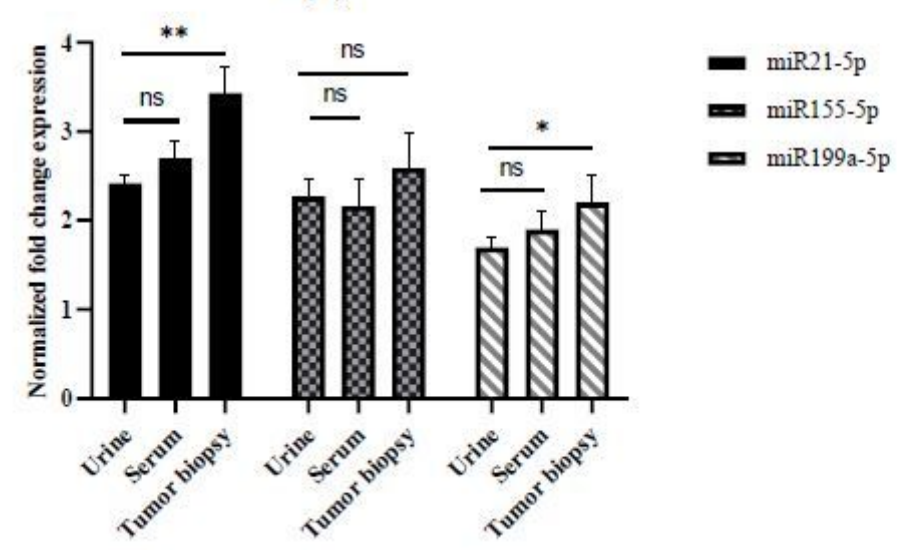

(b)

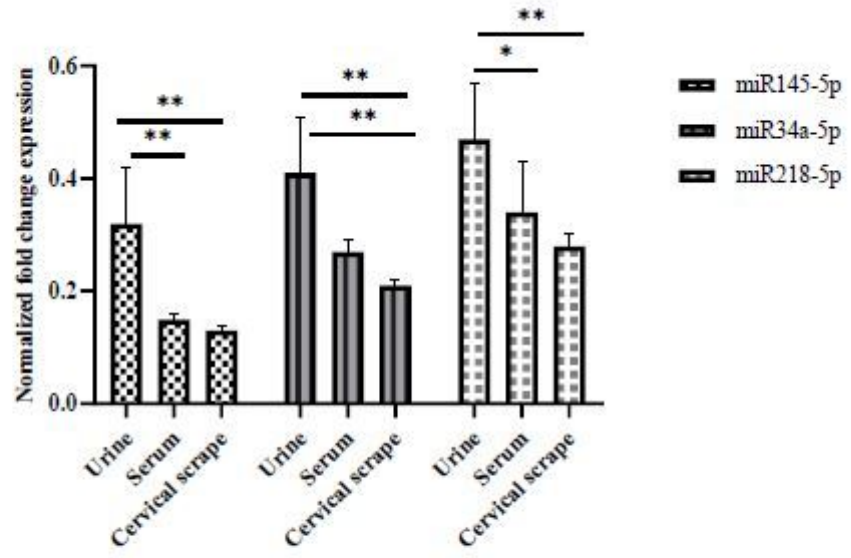

(d)

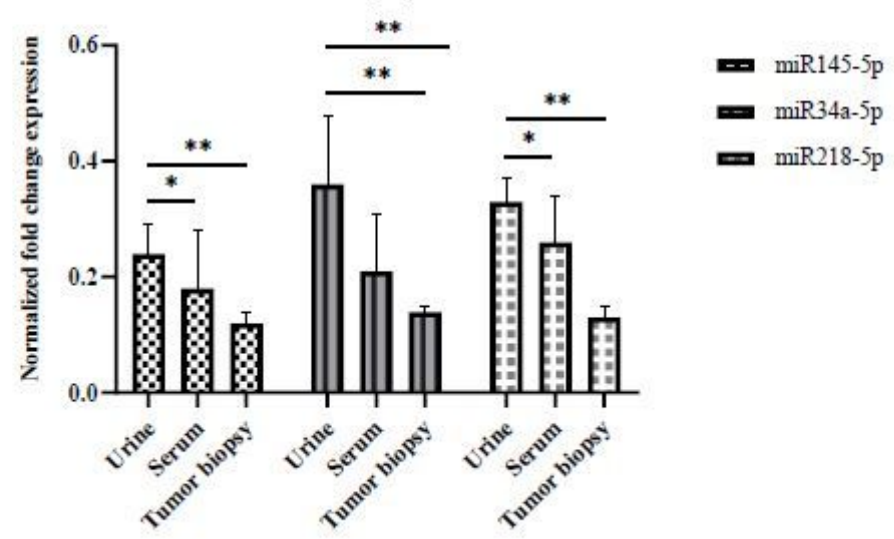

Figure 1

Comparative differential expression of six miRNAs in urine, serum, cervical scrape, and tissue biopsies derived from healthy controls, pre-cancer and cancer. (a \& c) The miRNA expression level of upregulated miRNAs (miR-21-5p, miR-155-5p, miR-199a-5p) and (b \& d) down regulated miRNAs (miR-145-5p, miR218 $-5 p$, and miR-34a-5p). Urine and serum samples were taken from pre-cancer and cervical cancer patients and compared to samples from healthy controls. In case of cervical scrape, the samples were derived from pre-cancer patients compared to samples from healthy volunteers, while in case of tissue biopsies, the samples were derived from cancer patient compared to samples from adjacent non-malignant tissues. ${ }^{*} \mathrm{p} \leq 0.01$, ns (nonsignificant). 
(a)

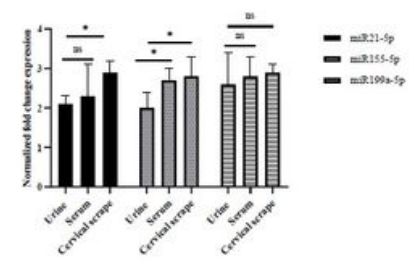

(e)

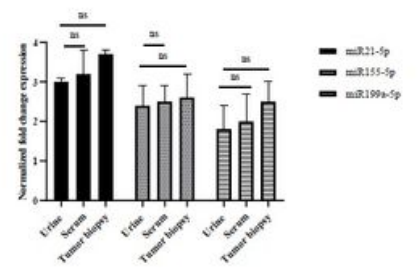

(b)

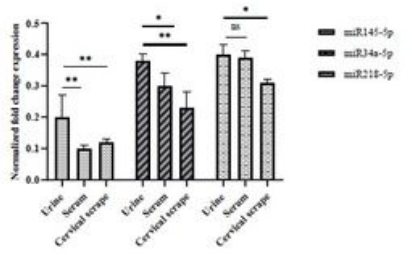

(f)

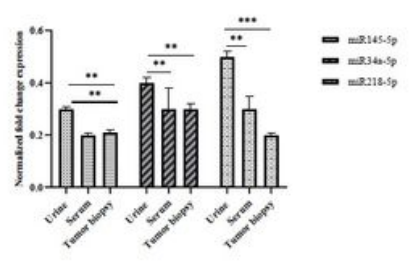

(c)
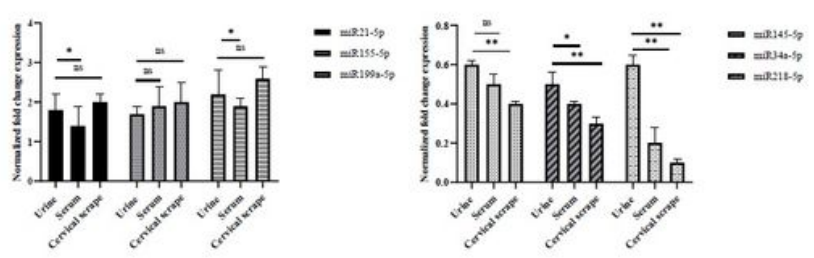

(g)

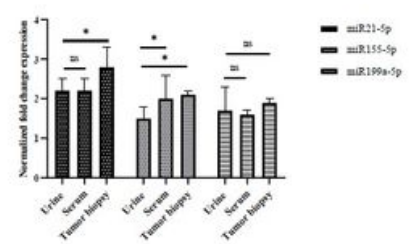

\section{Figure 2}

Expression levels of miR-21-5p, miR-155-5p, miR-199a-5p, miR-145-5p, miR-218-5p, and miR-34a-5p in HPV16-positive (a \& c) and HPV16-negative (b \& d) urine, serum, and cervical scrape samples of cervical pre-cancer and in HPV16-positive (e \& g) and HPV16-negative ( $\&$ h) urine, serum, and tissue biopsies of cervical cancer patients .

(a)

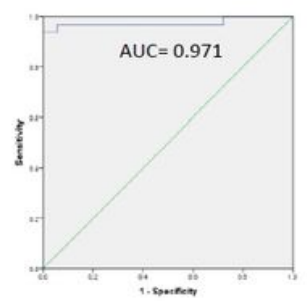

(f)

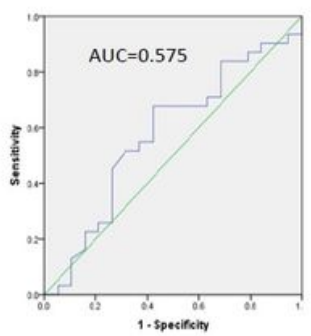

(b)

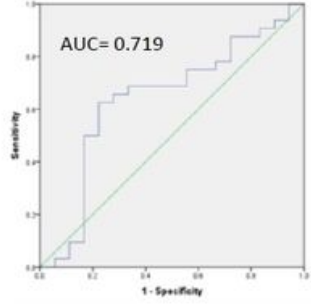

(g)

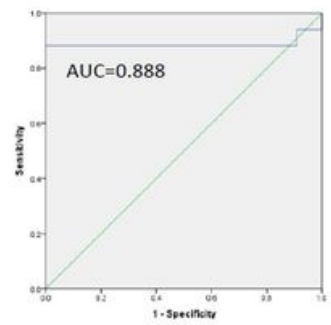

(c)

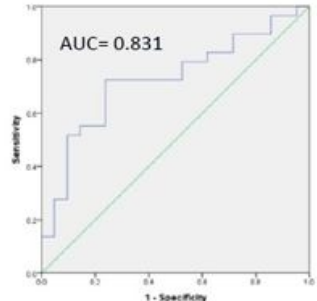

(h)

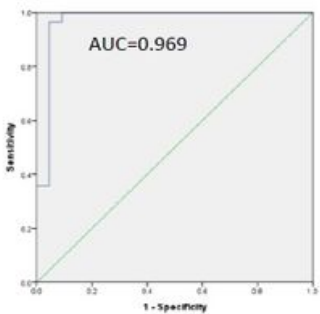

(d)

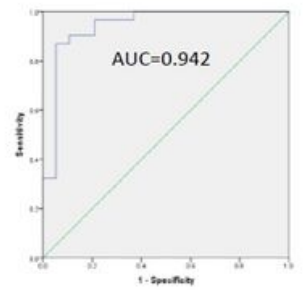

(i)

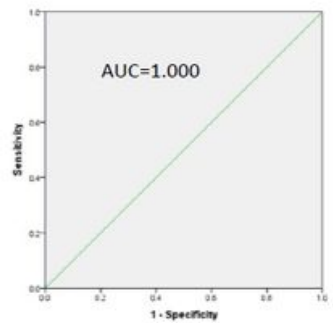

(e)

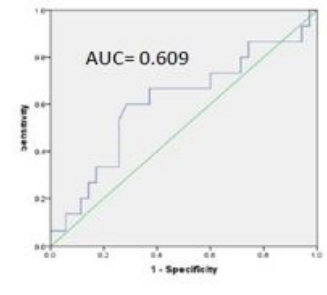




\section{Figure 3}

Receiver operating characteristics (ROC) plots of miRNA expression in urine sample of cervical cancer patients. ROC plots evaluating diagnostic potential of (a) miR-21-5p, (b) miR-155-5p, (c) miR-199a-5p, (d) combined ROC plots of three upregulated miRNAs (miR-21-5p, miR-155-5p, and miR-199a-5p), (e) miR218-5p, (f) miR-145-5p, (g) miR-34a-5p, (h) combined ROC plots of three downregulated miRNAs (miR218-5p, miR34a-5p, and miR-145-5p), and (I) combined ROC plots of all six miRNAs.

(a)

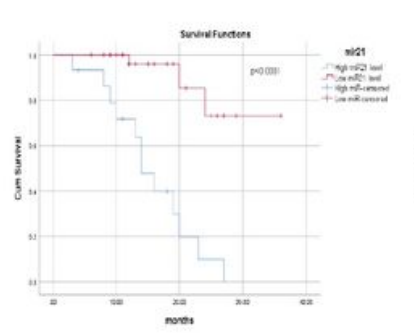

(e)

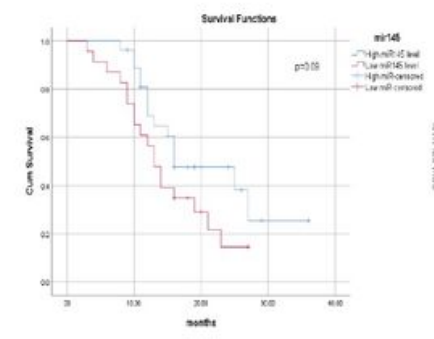

(b)

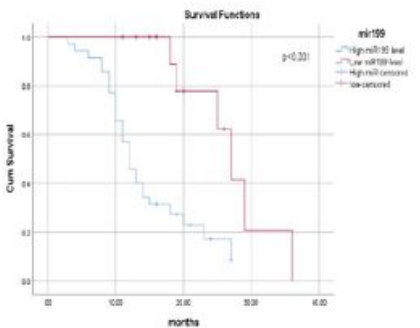

(f)

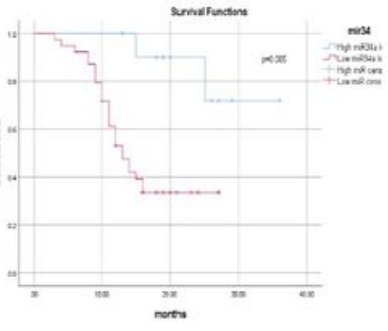

(c)

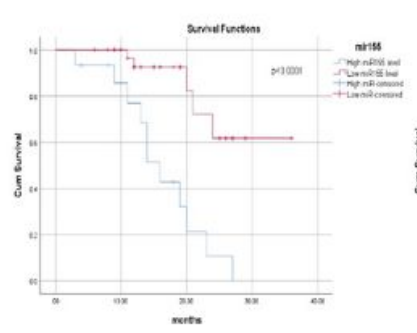

(g)

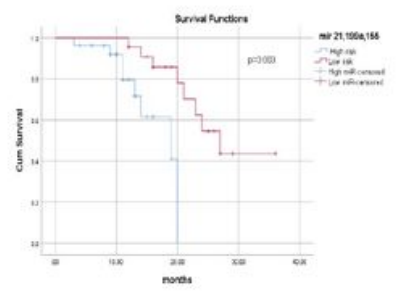

(d)

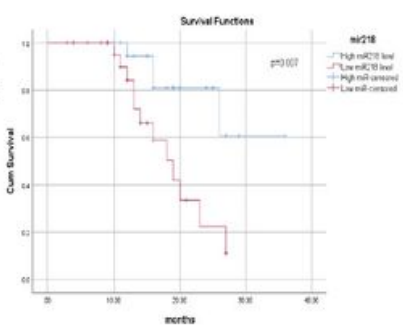

(h)

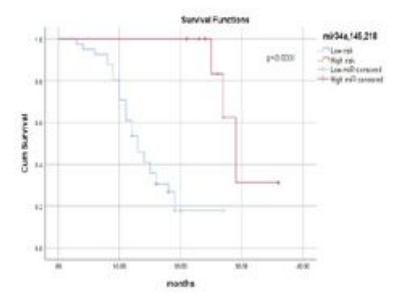

\section{Figure 4}

The correlation of miRNA expression with overall survival of cervical cancer patients calculated using Kaplan Meier curve and Log-rank test. The patients were stratified into high expression and low expression group according to median of each miRNA. (a) miR-21-5p, (b) miR-199a-5p, (c) miR-155-5p, (d) miR-218-5p, (e) miR-145-5p, (f) miR-34a-5p, (g) three upregulated miRNA signatures, and (h) three downregulated miRNA signatures. The patients were stratified into high risk group and low risk group based on median.

\section{Supplementary Files}

This is a list of supplementary files associated with this preprint. Click to download.

- SupplementaryFiguresTables.pdf 